\title{
The Elusive Quest to Maximize Voluntary Incident Reporting and Optimize Pharmacovigilance: A Matter of the Reporting Mechanisms or More?
}

\section{Sandra L. Kane-Gilli* and Lindsay N. McCartney²}

${ }^{1}$ Associate Professor of Pharmacy and Therapeutics, and Clinical Translational Science Institute, Center for Pharmacoinformatics and Outcomes Research, University of Pittsburgh School of Pharmacy, Critical Care Medication Safety Officer, Department of Pharmacy, University of Pittsburgh Medical Center, Pittsburgh, PA, USA ${ }^{2}$ Doctor of Pharmacy Candidate, University of Pittsburgh School of Pharmacy, Pittsburgh, PA, USA

Voluntary reporting is the primary method for medication error (ME) and adverse drug event (ADE) surveillance at most institutions. While other methods of event detection should be considered to optimize pharmacovigilance in an active surveillance system, voluntary reporting remains the cornerstone because of limited resources [1]. The dependence on voluntary reporting requires institutions to optimize the number of reports to understand current events and the possibility for future systematic changes [2]. Currently, there is not a recommended procedure for hospital personnel to voluntarily report suspected or proven errors. Two mechanisms for reporting have been used, traditional paper based and electronic systems.

It is suggested that manual, card-based reporting systems result in more frequent error reporting compared to electronic reporting programs. The observational study supporting this finding was conducted in an era of technological advancement where the use of electronic systems for voluntary reporting should be advocated [2]. Therefore, one must pose the question as to why a card-based reporting system was so successful and if this should be the preferred method over electronic voluntary reporting.

The card-based reporting system was conducted in critically ill patients in four phases, (1) education, (2) introduction of the cardbased system, known as SAFE [Safety, Action, Focus, Everyone], (3) withdrawal of the SAFE cards and reinstitution of the online reporting program, and (4) reinstitution of the SAFE cards. There were two additional, pre-existing reporting systems available for employees to voluntarily report errors throughout the study duration: the Risk Management Online Event/Incident Entry System (BJC Online) and the Pharmacy Resources Directory (PHRED) [3]. It was concluded that the use of the SAFE cards increased error reporting from healthcare workers over 2 -fold. In addition, physician reporting increased from 0.15 to 6.5 reported events/1,000 patient days.

Reflecting thoroughly upon the rationale for this positive outcome, there are several other factors that could have influenced reporting beyond the introduction of SAFE cards. The SAFE cards were promoted heavily in the institution with individual and group training sessions. A culture of patient safety existed through error reporting, quality improvements for the ICUs, and updating the hospital's electronic reporting system [3]. The SAFE cards provided optional anonymity in reporting. A safety specialist provided feedback to those who submitted an error and disclosed their identity. Also, the increase in physician reporting could be explained by the fact that the two previously used reporting systems, BJC Online and PHRED were restricted. The BJC Online system was mostly used by nurses. The majority of physicians had limited access or knowledge of the BJC online program because they were employed through a university, instead of the hospital and lacked the proper knowledge to utilize the program [3]. The PHRED system was only available for pharmacists to report medication errors and ADEs.
During phase 3 when SAFE cards were withdrawn, a renovated electronic reporting system was launched to mimic the SAFE card design, yet the total error reporting decreased during this time. If the electronic system was designed to match the SAFE cards, another variable of the study could explain the difference in use: the unequal emphasis on staff education during each phase of the project. There was no mention of a reminder or re-education process for how to properly report errors using the updated online system, the initial training of how new hospital workers reported errors, a description of how employees were trained to use the older-electronic reporting compared to the SAFE cards. Additionally, the authors did not mention if the hospital relied on paper or electronic medical charts. This factor could play into the culture of the institution. If physicians were already required to handwrite patient charts, a natural tendency would be to favor the SAFE cards. Nonetheless, this study highlights methods to improve voluntary reporting by educating employees on specific reporting programs, developing an anonymous system and providing feedback [3].

On the contrary, another study used electronic methods to improve voluntary reporting [4]. This study was conducted in three phases, (1) a planning process that instituted a new policy on non-punitive error reporting through paper based methods, (2) a transition period when a new electronic reporting system was implemented and the use of the paper system was still allowed, and (3) the full implementation of only the electronic system and removal of the paper-based. Education, upper management leadership support, and feedback to increase the reporting frequency for adverse clinical events were also included during phase 3. In addition, monthly newsletters were initiated updating staff on medical errors previously reported through their voluntary reporting system. The study demonstrated an increase in several areas of error reporting, including medication and adverse clinical events with the implementation of the electronic reporting system in phase 3.

Finally, the contributions of repetitive requests by asking physicians at patient discharge in the electronic medical record about medical errors during hospitalization seem to influence reporting improvement

*Corresponding author: Sandra L. Kane-Gill, Pharm.D., M.Sc., FCCM, 918 Salk Hall, 3501 Terrace St., Pittsburgh, PA 15261, USA, Tel: 412/624-5150; Fax: 412/6241850; E-mail: SLK54@pitt.edu

Received June 28, 2012; Accepted July 05, 2012; Published July 07, 2012

Citation: Kane-Gill SL, McCartney LN (2012) The Elusive Quest to Maximize Voluntary Incident Reporting and Optimize Pharmacovigilance: A Matter of the Reporting Mechanisms or More? Adv Pharmacoepidem Drug Safety 1:e108. doi:10.4172/2167-1052.1000e108

Copyright: @ 2012 Kane-Gill SL, et al. This is an open-access article distributed under the terms of the Creative Commons Attribution License, which permits unrestricted use, distribution, and reproduction in any medium, provided the original author and source are credited. 
Citation: Kane-Gill SL, McCartney LN (2012) The Elusive Quest to Maximize Voluntary Incident Reporting and Optimize Pharmacovigilance: A Matter of the Reporting Mechanisms or More? Adv Pharmacoepidem Drug Safety 1:e108. doi:10.4172/2167-1052.1000e108

Page 2 of 2

[5]. This process provided a convenient and quick electronic method for physicians to report errors. Again, this institutional change was not completed in isolation, a new position, the "Team Safety Officer" was initiated, a weekly Safety Report was developed and incentives for the best catch of each month was provided.

These studies illustrate that voluntary incident reporting can be maximized to improve pharmacovigilance with education and program awareness. Specific actions to be taken to improve reporting include: incorporating individual and group education, providing individual and group feedback, providing optional anonymity for reporting, providing incentives for reporting, establishing a culture of safety, updating policies/procedures for reporting, and adding hospital safety employees. Therefore, it is by incorporating these techniques that voluntary error reporting can be improved in the hospital settings, not necessarily by focusing on the mechanism for reporting.

\section{References}

1. Kane-Gill SL, Devlin JW (2006) Adverse drug event reporting in intensive care units: a survey of current practices. Ann Pharmacother 40: 1267-1273.

2. Stockwell DC, Kane-Gill SL (2010) Developing a patient safety surveillance system to identify adverse events in the intensive care unit. Crit Care Med 38: S117-S125.

3. Harris CB, Krauss MJ, Coopersmith CM, Avidan M, Nast PA, et al. (2007) Patient safety event reporting in critical care: A study of three intensive care units. Crit Care Med 35: 1068-1076.

4. Tuttle D, Holloway R, Baird T, Sheehan B, Skelton WK (2004) Electronic reporting to improve patient safety. QualSaf Health Care 13: 281-286.

5. Foster PN, Sidhu R, Gadhia DA, DeMusis M (2008) Leveraging computerized sign-out to increase error reporting and addressing patient safety in graduate medical education. J Gen Intern Med 23: 481- 484. 